\title{
ON GENERALIZED EIGHTH ORDER MOCK THETA FUNCTIONS
}

\author{
Pramod Kumar Rawat \\ University of Lucknow, Lucknow 226007, India \\ pramodkrawat@yahoo.com
}

\begin{abstract}
In this paper we have generalized eighth order mock theta functions, recently introduced by Gordon and MacIntosh involving four independent variables. The idea of generalizing was to have four extra parameters, which on specializing give known functions and thus these results hold for those known functions. We have represented these generalized functions as $q$-integral. Thus on specializing we have the classical mock theta functions represented as $q$-integral. The same is true for the multibasic expansion given.
\end{abstract}

Keywords: $q$-Hypergeometric Series, Mock Theta functions, Continued Fractions, $q$-Integrals.

\section{Introduction}

The last gift to mathematics by Ramanujan was mock theta functions. In his last letter to Hardy [5], Ramanujan introduced 17 functions and called them mock theta functions as they were not theta functions and classified them as 4 functions of third order, 10 functions of fifth order and 3 functions of seventh order though Ramanujan did not say what he meant by "order" of mock theta function. Later Watson [12] introduced 3 more mock theta functions of third order. Gordon and McIntosh [7] gave eight more mock theta functions and called them of eighth order. Andrews and Hickerson [3] said the "order" is connected with combinatorics interpretation. Andrews [1] generalized five third order mock theta functions. Srivastava [11] generalized eighth order mock theta function. Recently Choi [4] also generalized mock theta functions of third, fifth, sixth, seventh and tenth order.

Motivated by Andrews' generalization of five of seven third order mock theta functions and Choi's generalization, we have tried to generalize the eighth order mock theta functions by introducing four independent variables. The advantage is that by specializing the parameters we can have known functions.

In this paper we have represented these generalized functions as $q$-integral and we have also given the multibasic expansion. Thus we have on specializing the parameters, the classical mock theta functions representation as $q$-integral and the multibasic expansion for generalized functions reduced to classical mock theta function of eighth order.

\section{Definitions and notations}

The eighth order mock theta functions of Gordon and Mclntosh [7] are

$$
\begin{gathered}
S_{0}(q)=\sum_{n=0}^{\infty} \frac{q^{n^{2}}\left(-q ; q^{2}\right)_{n}}{\left(-q^{2} ; q^{2}\right)_{n}}, \quad S_{1}(q)=\sum_{n=0}^{\infty} \frac{q^{n(n+2)}\left(-q ; q^{2}\right)_{n}}{\left(-q^{2} ; q^{2}\right)_{n}}, \\
T_{0}(q)=\sum_{n=0}^{\infty} \frac{q^{(n+1)(n+2)}\left(-q^{2} ; q^{2}\right)_{n}}{\left(-q ; q^{2}\right)_{n+1}}, \quad T_{1}(q)=\sum_{n=0}^{\infty} \frac{q^{n^{2}+n}\left(-q^{2} ; q^{2}\right)_{n}}{\left(-q ; q^{2}\right)_{n+1}},
\end{gathered}
$$




$$
\begin{gathered}
U_{0}(q)=\sum_{n=0}^{\infty} \frac{q^{n^{2}}\left(-q ; q^{2}\right)_{n}}{\left(-q^{4} ; q^{4}\right)_{n}}, \quad U_{1}(q)=\sum_{n=0}^{\infty} \frac{q^{(n+1)^{2}}\left(-q ; q^{2}\right)_{n}}{\left(-q^{2} ; q^{4}\right)_{n+1}} \\
V_{0}(q)=-1+2 \sum_{n=0}^{\infty} \frac{q^{n^{2}}\left(-q ; q^{2}\right)_{n}}{\left(q ; q^{2}\right)_{n}}=-1+2 \sum_{n=0}^{\infty} \frac{q^{2 n^{2}}\left(-q^{2} ; q^{4}\right)_{n}}{\left(q ; q^{2}\right)_{2 n+1}} \\
V_{1}(q)=\sum_{n=0}^{\infty} \frac{q^{(n+1)^{2}}\left(-q ; q^{2}\right)_{n}}{\left(q ; q^{2}\right)_{n+1}}=\sum_{n=0}^{\infty} \frac{q^{2 n^{2}+2 n+1}\left(-q^{4} ; q^{4}\right)_{n}}{\left(q ; q^{2}\right)_{2 n+2}}
\end{gathered}
$$

where

$$
\left(a ; q^{k}\right)_{n}=\prod_{j=1}^{n}\left(1-a q^{k(j-1)}\right),\left(a ; q^{k}\right)_{\infty}=\prod_{j=1}^{\infty}\left(1-a q^{k(j-1)}\right), \quad \text { and } \quad\left(a ; q^{k}\right)_{0}=1
$$

\section{Generalized eighth order mock theta functions}

The four variable generalization of the eighth order mock theta functions are

$$
\begin{gathered}
S_{0}(t, \alpha, \beta, z ; q)=\frac{1}{(t)_{\infty}} \sum_{n=0}^{\infty} \frac{(t)_{n} q^{n^{2}-n+n \beta}\left(-z q ; q^{2}\right)_{n} \alpha^{n}}{\left(-\alpha q^{2} ; q^{2}\right)_{n}}, \\
T_{0}(t, \alpha, \beta, z ; q)=\frac{1}{(t)_{\infty}} \sum_{n=0}^{\infty} \frac{(t)_{n} q^{n^{2}+3 n+2-n+n \beta}\left(-q^{2} / \alpha ; q^{2}\right)_{n}}{\left(-q / z ; q^{2}\right)_{n+1} z^{n+1}}, \\
U_{0}(t, \alpha, \beta, z ; q)=\frac{1}{(t)_{\infty}} \sum_{n=0}^{\infty} \frac{(t)_{n} q^{n^{2}-n+n \beta}\left(-z q ; q^{2}\right)_{n} \alpha^{n}}{\left(-\alpha q^{4} ; q^{4}\right)_{n}} \\
V_{0}(t, \alpha, \beta, z ; q)=-1+\frac{1}{(t)_{\infty}} \sum_{n=0}^{\infty} \frac{(t)_{n} q^{n^{2-n+n \beta}}\left(-z q ; q^{2}\right)_{n} \alpha^{n}}{\left(\alpha q ; q^{2}\right)_{n}} \\
S_{1}(t, \alpha, \beta, z ; q)=\frac{1}{(t)_{\infty}} \sum_{n=0}^{\infty} \frac{(t)_{n} q^{n^{2}+2 n-n+n \beta}\left(-z q ; q^{2}\right)_{n} \alpha^{n}}{\left(-\alpha q^{2} ; q^{2}\right)_{n}} \\
T_{1}(t, \alpha, \beta, z ; q)=\frac{1}{(t)_{\infty}} \sum_{n=0}^{\infty} \frac{(t)_{n} q^{n^{2}+n-n+n \beta}\left(-q^{2} / \alpha ; q^{2}\right)_{n}}{\left(-q / z ; q^{2}\right)_{n+1} z^{n+1}} \\
U_{1}(t, \alpha, \beta, z ; q)=\frac{1}{(t)_{\infty}} \sum_{n=0}^{\infty} \frac{(t)_{n} q^{(n+1)^{2}-n+n \beta}\left(-z q ; q^{2}\right)_{n}}{\left(-\alpha q^{2} ; q^{4}\right)_{n+1}} \\
V_{1}(t, \alpha, \beta, z ; q)=\frac{1}{(t)_{\infty}} \sum_{n=0}^{\infty} \frac{(t)_{n} q^{(n+1)^{2}-n+n \beta}\left(-z q ; q^{2}\right)_{n} \alpha^{n}}{\left(\alpha q ; q^{2}\right)_{n+1}} .
\end{gathered}
$$

For $t=0, \alpha=1, \beta=1$ and $z=1$ these functions reduce to classical mock theta functions.

\section{Relation between generalized eighth order mock theta functions}

The differential operator $D_{q}[8]$ is defined as

$$
z D_{q, z} F(z, \alpha)=F(z, \alpha)-F(z q, \alpha) .
$$

By using the differential operator we shall connect the generalized eighth order mock theta functions. 
Proposition 1. The following is true:

$$
\begin{aligned}
& \text { (i) } D_{q, t}^{2} S_{0}(t, \alpha, \beta, z ; q)=S_{1}(t, \alpha, \beta, z ; q), \\
& \text { (ii) } q^{2} D_{q, t}^{2} T_{1}(t, \alpha, \beta, z ; q)=T_{0}(t, z, \alpha, \beta, z ; q) .
\end{aligned}
$$

P r o o f. Proof of (i):

$$
\begin{gathered}
t D_{q, t} S_{0}(t, \alpha, \beta, z ; q)=\frac{1}{(t)_{\infty}} \sum_{n=0}^{\infty} \frac{(t)_{n} q^{n^{2}-n+n \beta}\left(-z q ; q^{2}\right)_{n} \alpha^{n}}{\left(-\alpha q^{2} ; q^{2}\right)_{n}}-\frac{1}{(t q)_{\infty}} \sum_{n=0}^{\infty} \frac{(t q)_{n} q^{n^{2}-n+n \beta}\left(-z q ; q^{2}\right)_{n} \alpha^{n}}{\left(-\alpha q^{2} ; q^{2}\right)_{n}} \\
=\frac{1}{(t)_{\infty}} \sum_{n=0}^{\infty} \frac{(t)_{n} q^{n^{2}-n+n \beta}\left(-z q ; q^{2}\right)_{n} \alpha^{n}}{\left(-\alpha q^{2} ; q^{2}\right)_{n}}-\frac{1}{(t)_{\infty}} \sum_{n=0}^{\infty} \frac{(t)_{n} q^{n^{2}-n+n \beta}\left(-z q ; q^{2}\right)_{n} \alpha^{n}}{\left(-\alpha q^{2} ; q^{2}\right)_{n}}\left(1-t q^{n}\right) \\
=\frac{t}{(t)_{\infty}} \sum_{n=0}^{\infty} \frac{(t)_{n} q^{n^{2}+n \beta}\left(-z q ; q^{2}\right)_{n} \alpha^{n}}{\left(-\alpha q^{2} ; q^{2}\right)_{n}} .
\end{gathered}
$$

Similarly

$$
\begin{aligned}
& D_{q, t^{2}}^{2} S_{0}(t, \alpha, \beta, z ; q)=\frac{1}{(t)_{\infty}} \sum_{n=0}^{\infty} \frac{(t)_{n} q^{n^{2}+n+n \beta}\left(-z q ; q^{2}\right)_{n} \alpha^{n}}{\left(-\alpha q^{2} ; q^{2}\right)_{n}} \\
= & \frac{1}{(t)_{\infty}} \sum_{n=0}^{\infty} \frac{(t)_{n} q^{n^{2}+2 n-n+n \beta}\left(-z q ; q^{2}\right)_{n} \alpha^{n}}{\left(-\alpha q^{2} ; q^{2}\right)_{n}}=S_{1}(t, z, \alpha, \beta ; q),
\end{aligned}
$$

which proves (i).

Proof of (ii):

$$
D_{q, t} T_{1}(t, \alpha, \beta, z ; q)=\frac{1}{(t)_{\infty}} \sum_{n=0}^{\infty} \frac{q^{n^{2}+2 n-n+n \beta}\left(-q^{2} / \alpha ; q^{2}\right)_{n}}{\left(-q / z ; q^{2}\right)_{n+1} z^{n+1}}
$$

and

$$
\begin{gathered}
D_{q, t}^{2} T_{1}(t, \alpha, \beta, z ; q)=\frac{1}{(t)_{\infty}} \sum_{n=0}^{\infty} \frac{q^{n^{2}+3 n-n+n \beta}\left(-q^{2} / \alpha ; q^{2}\right)_{n}}{\left(-q / z ; q^{2}\right)_{n+1} z^{n+1}}, \\
q^{2} D_{q, t}^{2} T_{1}(t, \alpha, \beta, z ; q)=\frac{1}{(t)_{\infty}} \sum_{n=0}^{\infty} \frac{q^{n^{2}+3 n+2-n+n \beta}\left(-q^{2} / \alpha ; q^{2}\right)_{n}}{\left(-q / z ; q^{2}\right)_{n+1} z^{n+1}}=T_{0}(t, \alpha, \beta, z ; q),
\end{gathered}
$$

which proves (ii).

\section{5. $q$-Integral representation for the generalized eighth order mock theta functions}

Thomae and Jackson [6, p. 19] defined $q$-integral

$$
\int_{0}^{1} f(t) d_{q} t=(1-q) \sum_{n=0}^{\infty} f\left(q^{n}\right) q^{n}
$$

using limiting case of $q$-beta integral, we have

$$
\frac{1}{\left(q^{x} ; q\right)_{\infty}}=\frac{(1-q)^{-1}}{(q ; q)_{\infty}} \int_{0}^{1} t^{x-1}(t q ; q)_{\infty} d_{q} t
$$

We now represent these generalized functions as $q$-integral. By specializing the parameters we have the integral representation for classical mock theta functions. 


\section{Theorem 1.}

$$
\begin{aligned}
\text { (i) } S_{0}\left(q^{t}, \alpha, \beta, z ; q\right) & =\frac{(1-q)^{-1}}{(q ; q)_{\infty}} \int_{0}^{1} u^{t-1}(u q ; q)_{\infty} S_{0}(0, \alpha, p u, z ; q) d_{q} u \\
\text { (ii) } T_{0}\left(q^{t}, \alpha, \beta, z ; q\right) & =\frac{(1-q)^{-1}}{(q ; q)_{\infty}} \int_{0}^{1} u^{t-1}(u q ; q)_{\infty} T_{0}(0, \alpha, p u, z ; q) d_{q} u \\
\text { (iii) } \quad U_{0}\left(q^{t}, \alpha, \beta, z ; q\right) & =\frac{(1-q)^{-1}}{(q ; q)_{\infty}} \int_{0}^{1} u^{t-1}(u q ; q)_{\infty} U_{0}(0, \alpha, p u, z ; q) d_{q} u \\
\text { (iv) } \quad V_{0}\left(q^{t}, \alpha, \beta, z ; q\right) & =\frac{(1-q)^{-1}}{(q ; q)_{\infty}} \int_{0}^{1} u^{t-1}(u q ; q)_{\infty} V_{0}(0, \alpha, p u, z ; q) d_{q} u \\
\text { (v) } S_{1}\left(q^{t}, \alpha, \beta, z ; q\right) & =\frac{(1-q)^{-1}}{(q ; q)_{\infty}} \int_{0}^{1} u^{t-1}(u q ; q)_{\infty} S_{1}(0, \alpha, p u, z ; q) d_{q} u \\
\text { (vi) } \quad T_{1}\left(q^{t}, \alpha, \beta, z ; q\right) & =\frac{(1-q)^{-1}}{(q ; q)_{\infty}} \int_{0}^{1} u^{t-1}(u q ; q)_{\infty} T_{1}(0, \alpha, p u, z ; q) d_{q} u \\
\text { (vii) } \quad U_{1}\left(q^{t}, \alpha, \beta, z ; q\right) & =\frac{(1-q)^{-1}}{(q ; q)_{\infty}} \int_{0}^{1} u^{t-1}(u q ; q)_{\infty} U_{1}(0, \alpha, p u, z ; q) d_{q} u \\
\text { (viii) } V_{1}\left(q^{t}, \alpha, \beta, z ; q\right) & =\frac{(1-q)^{-1}}{(q ; q)_{\infty}} \int_{0}^{1} u^{t-1}(u q ; q)_{\infty} V_{1}(0, \alpha, p u, z ; q) d_{q} u
\end{aligned}
$$

P r o o f. A detailed proof for $S_{0}\left(q^{t}, \alpha, \beta, z ; q\right)$ is given. The proofs of the other functions are similar, so omitted.

Proof of (i): By definition

$$
S_{0}(t, \alpha, \beta, z ; q)=\frac{1}{(t)_{\infty}} \sum_{n=0}^{\infty} \frac{(t)_{n} q^{n^{2}-n+n \beta}\left(-z q ; q^{2}\right)_{n} \alpha^{n}}{\left(-\alpha q^{2} ; q^{2}\right)_{n}} .
$$

Replacing $t$ by $q^{t}$, we have

$$
\begin{gathered}
S_{0}\left(q^{t}, \alpha, \beta, z ; q\right)=\frac{1}{\left(q^{t}\right)_{\infty}} \sum_{n=0}^{\infty} \frac{\left(q^{t}\right)_{n} q^{n^{2}-n+n \beta}\left(-z q ; q^{2}\right)_{n} \alpha^{n}}{\left(-\alpha q^{2} ; q^{2}\right)_{n}}=\sum_{n=0}^{\infty} \frac{q^{n^{2}-n+n \beta}\left(-z q ; q^{2}\right)_{n} \alpha^{n}}{\left(-\alpha q^{2} ; q^{2}\right)_{n}\left(q^{n+t} ; q\right)_{\infty}} \\
=\sum_{n=0}^{\infty} \frac{q^{n^{2}-n+n \beta}\left(-z q ; q^{2}\right)_{n} \alpha^{n}}{\left(-\alpha q^{2} ; q^{2}\right)_{n}} \frac{(1-q)^{-1}}{(q ; q)_{\infty}} \int_{0}^{1} u^{t-1}(u q ; q)_{\infty} d_{q} u
\end{gathered}
$$

but

$$
S_{0}(0, \alpha, \beta, z ; q)=\sum_{n=0}^{\infty} \frac{q^{n^{2}-n+n \beta}\left(-z q ; q^{2}\right)_{n} \alpha^{n}}{\left(-\alpha q^{2} ; q^{2}\right)_{n}}
$$

putting $q^{\beta}=p$, we have

$$
\begin{gathered}
S_{0}(0, \alpha, p, z ; q)=\sum_{n=0}^{\infty} \frac{q^{n^{2}-n}\left(-z q ; q^{2}\right)_{n} \alpha^{n} p^{n}}{\left(-\alpha q^{2} ; q^{2}\right)_{n}} \\
S_{0}\left(q^{t}, \alpha, \beta, z ; q\right)=\frac{(1-q)^{-1}}{(q ; q)_{\infty}} \int_{0}^{1} u^{t-1}(u q ; q)_{\infty} S_{0}(0, \alpha, p u, z ; q) d_{q} u,
\end{gathered}
$$

which proves (i).

The proof of all the other functions is similar. Taking $\alpha=1, \beta=1$ and $z=1$ we have the integral representation of the classical eighth order mock theta functions. 


\section{Multibasic expansion of generalized eighth order mock theta functions}

The following bibasic expansion will be used to give multibasic expansion for the generalized functions.

Theorem 2. The following is true:

$$
\begin{gathered}
\sum_{k=0}^{\infty} \frac{\left(1-a p^{k} q^{k}\right)\left(1-b p^{k} q^{-k}\right)(a, b ; p)_{k}(c, a / b c ; q)_{k} q^{k}}{(1-a)(1-b)(q, a q / b ; q)_{k}(a p / c, b c p ; p)_{k}} \sum_{m=0}^{\infty} \alpha_{m+k} \\
=\sum_{m=0}^{\infty} \frac{(a p, b p ; p)_{m}(c q, a q / b c ; q)_{m} q^{m}}{(q, a q / b ; q)_{m}(a p / c, b c p ; p)_{m}} \alpha_{m} .
\end{gathered}
$$

P r o o f. Using the summation formula [6, (3.6.7), p. 71] we have

$$
\begin{gathered}
\sum_{k=0}^{n} \frac{\left(1-a p^{k} q^{k}\right)\left(1-b p^{k} q^{-k}\right)}{(1-a)(1-b)} \frac{(a, b ; p)_{k}(c, a / b c ; q)_{k}}{(q, a q / b ; q)_{k}(a p / c, b c p ; p)_{k}} q^{k} \\
=\frac{(a p, b p ; p)_{n}(c q, a q / b c ; q)_{n}}{(q, a q / b ; q)_{n}(a p / c, b c p ; p)_{n}}
\end{gathered}
$$

and [9, Lemma 10, p. 57],

$$
\sum_{n=0}^{\infty} \sum_{k=0}^{n} B(k, n)=\sum_{n=0}^{\infty} \sum_{k=0}^{\infty} B(k, n+k),
$$

therefore we get the statement of the theorem.

We will consider the following case of Theorem 2 .

Case I. Letting $q \rightarrow q^{3}$ and $c \rightarrow \infty$ in Theorem 1 , we have

$$
\begin{gathered}
\sum_{k=0}^{\infty} \frac{\left(1-a p^{k} q^{3 k}\right)\left(1-b p^{k} q^{-3 k}\right)(a, b ; p)_{k} q^{\left(3 k^{2}+3 k\right) / 2}}{(1-a)(1-b)\left(q^{3}, a q^{3} / b ; q^{3}\right)_{k} b^{k} p^{\left(k^{2}+k\right) / 2}} \sum_{m=0}^{\infty} \alpha_{m+k} \\
=\sum_{m=0}^{\infty} \frac{(a p, b p ; p)_{m} q^{\left(3 m^{2}+3 m\right) / 2}}{\left(q^{3}, a q^{3} / b ; q^{3}\right)_{m} b^{m} p^{\left(m^{2}+m\right) / 2}} \alpha_{m}
\end{gathered}
$$

Theorem 3. The multibasic hypergeometric expansion of these generalized functions are:

(i) $S_{0}(t, \alpha, 1, z ; q)=\frac{1}{(t)_{\infty}} \sum_{k=0}^{\infty} \frac{\left(1-t q^{4 k-1}\right)\left(1-k^{-2 k+2}\right)(t ; q)_{k-1}\left(-z q ; q^{2}\right)_{k} q^{k^{2}} \alpha^{k}}{\left(1-q^{k+2}\right)\left(-\alpha q^{2} ; q^{2}\right)_{k}}$

$$
\times \phi\left[\begin{array}{l}
q ;-z q^{2 k+1} ; t q^{3 k}, q^{3 k+3} \\
q^{k+3} ;-\alpha q^{2 k+2}: 0
\end{array} ; q, q^{2}, q^{3} ; q \alpha\right]
$$

(ii) $T_{0}(t, \alpha, 1, z ; q)=\frac{1}{(t)_{\infty}} \sum_{k=0}^{\infty} \frac{\left(1-t q^{4 k-1}\right)\left(1-k^{-2 k+2}\right)(t ; q)_{k-1}\left(-q^{2} / \alpha ; q^{2}\right)_{k} q^{k^{2}+3 k+2} \alpha^{k}}{\left(1-q^{k+2}\right)\left(-q / z ; q^{2}\right)_{k+1} z^{k+1}}$

$$
\times \phi\left[\begin{array}{l}
q ;-q^{2 k+2} / \alpha ; t q^{3 k}, q^{3 k+3} \\
q^{k+3} ;-q^{2 k+2} / z: 0
\end{array} ;, q^{2}, q^{3} ; q^{4} \alpha\right],
$$

(iii) $\quad U_{0}(t, \alpha, 1, z ; q)=\frac{1}{(t)_{\infty}} \sum_{k=0}^{\infty} \frac{\left(1-t q^{4 k-1}\right)\left(1-k^{-2 k+2}\right)(t ; q)_{k-1}\left(-z q ; q^{2}\right)_{k} q^{k^{2}} \alpha^{k} z^{2 k}}{\left(1-q^{k+2}\right)\left(-\alpha q^{4} ; q^{4}\right)_{k}}$

$$
\times \phi\left[\begin{array}{l}
q ;-z q^{2 k+1} ; t q^{3 k}, q^{3 k+3} ; 0 \\
q^{k+3} ; 0 ; 0 ;-\alpha q^{4 k+4}
\end{array} ;, q^{2}, q^{3}, q^{4} ; z^{2} q \alpha\right],
$$


(iv) $V_{0}(t, \alpha, 1, z ; q)=\frac{-1}{(t)_{\infty}}+\frac{1}{(t)_{\infty}} \sum_{k=0}^{\infty} \frac{\left(1-t q^{4 k-1}\right)\left(1-k^{-2 k+2}\right)(t ; q)_{k-1}\left(-z q ; q^{2}\right)_{k} q^{k^{2}} \alpha^{k} z^{2 k}}{\left(1-q^{k+2}\right)\left(\alpha q ; q^{2}\right)_{k}}$ $\times \phi\left[\begin{array}{l}q ;-z q^{2 k+1} ; q^{3 k}, q^{3 k+3} \\ q^{k+3} ;-\alpha q^{2 k+1}: 0\end{array} ;, q^{2}, q^{3} ; q \alpha z^{2}\right]$,

(v) $S_{1}(t, \alpha, 1, z ; q)=\frac{1}{(t)_{\infty}} \sum_{k=0}^{\infty} \frac{\left(1-t q^{4 k-1}\right)\left(1-k^{-2 k+2}\right)(t ; q)_{k-1}\left(-z q ; q^{2}\right)_{k} q^{k^{2}+2 k} \alpha^{k}}{\left(1-q^{k+2}\right)\left(-\alpha q^{2} ; q^{2}\right)_{k}}$ $\times \phi\left[\begin{array}{l}q ;-z q^{2 k+1} ; t q^{3 k}, q^{3 k+3} \\ q^{k+3} ;-\alpha q^{2 k+2}: 0\end{array} ;, q^{2}, q^{3} ; q^{3} \alpha\right]$,

(vi) $T_{1}(t, \alpha, 1, z ; q)=\frac{1}{(t)_{\infty}} \sum_{k=0}^{\infty} \frac{\left(1-t q^{4 k-1}\right)\left(1-k^{-2 k+2}\right)(t ; q)_{k-1}\left(-q^{2} / \alpha ; q^{2}\right)_{k} q^{k^{2}+k} \alpha^{k}}{\left(1-q^{k+2}\right)\left(-q / z ; q^{2}\right)_{k+1} z^{k+1}}$ $\times \phi\left[\begin{array}{l}q ;-q^{2 k+2} / \alpha ; t q^{3 k}, q^{3 k+3} \\ q^{k+3} ;-q^{2 k+3} / z: 0\end{array} ;, q^{2}, q^{3} ; q^{2} z^{-1} \alpha\right]$,

(vii) $\quad U_{1}(t, \alpha, 1, z ; q)=\frac{1}{(t)_{\infty}} \sum_{k=0}^{\infty} \frac{\left(1-t q^{4 k-1}\right)\left(1-k^{-2 k+2}\right)(t ; q)_{k-1}\left(-z q ; q^{2}\right)_{k} q^{(k+1)^{2}} \alpha^{k} z^{2 k}}{\left(1-q^{k+2}\right)\left(-\alpha q^{2} ; q^{4}\right)_{k+1}}$ $\times \phi\left[\begin{array}{l}q ;-z q^{2 k+1} ; t q^{3 k}, q^{3 k+3} \\ q^{k+3} ; 0 ; 0 ;-\alpha q^{4 k+6}: 0\end{array} ; q, q^{2}, q^{3}, q^{4} ; q^{3} z^{2} \alpha\right]$

(viii) $\quad V_{1}(t, \alpha, 1, z ; q)=\frac{1}{(t)_{\infty}} \sum_{k=0}^{\infty} \frac{\left(1-t q^{4 k-1}\right)\left(1-k^{-2 k+2}\right)(t ; q)_{k-1}\left(-z q ; q^{2}\right)_{k} q^{(k+1)^{2}} \alpha^{k} z^{2 k}}{\left(1-q^{k+2}\right)\left(\alpha q ; q^{2}\right)_{k+1}}$ $\times \phi\left[\begin{array}{l}q ;-z q^{2 k+1} ; t q^{3 k}, q^{3 k+3} \\ q^{k+3} ; \alpha q^{2 k+3}: 0\end{array} ; q, q^{2}, q^{3} ; q^{3} z^{2} \alpha\right]$.

P r o o f. We shall give the proof of (i) only, for others we will state the value of parameters.

Proof of (i): Taking $a=t / q, b=q^{2}, p=q$ and

$$
\alpha_{m}=\frac{\left(q^{3} ; q^{3}\right)_{m}\left(t ; q^{3}\right)_{m}\left(-z q ; q^{2}\right)_{m} \alpha^{m} q^{m}}{\left(q^{3} ; q\right)_{m}\left(-\alpha q^{2} ; q^{2}\right)_{m}} \text { in (6.2), }
$$

we have

$$
\begin{gathered}
\sum_{k=0}^{\infty} \frac{\left(1-t q^{4 k-1}\right)\left(1-q^{-2 k+2}\right)\left(t / q, q^{2} ; q\right)_{k} q^{k^{2}+k}}{(1-t / q)\left(1-q^{2}\right)\left(q^{3}, t ; q^{3}\right)_{k} q^{2 k}} \\
\times \sum_{m=0}^{\infty} \frac{\left(t ; q^{3}\right)_{m+k}\left(q^{3} ; q^{3}\right)_{m+k}\left(-z q ; q^{2}\right)_{m+k} \alpha^{m+k} q^{m+k}}{\left(q^{3} ; q\right)_{m+k}\left(-\alpha q^{2} ; q^{2}\right)_{m+k}} \\
=\sum_{m=0}^{\infty} \frac{\left(t, q^{3} ; q\right)_{m} q^{m^{2}+m}}{\left(q^{3}, t ; q^{3}\right)_{m} q^{2 m}} \frac{\left(q^{3} ; q^{3}\right)_{m}\left(t ; q^{3}\right)_{m}\left(-z q ; q^{2}\right)_{m} \alpha^{m} q^{m}}{\left(q^{3} ; q\right)_{m}\left(-\alpha q^{2} ; q^{2}\right)_{m}} .
\end{gathered}
$$

The right hand side is equal to

$$
\begin{aligned}
& \sum_{m=0}^{\infty} \frac{\left(t, q^{3} ; q\right)_{m} q^{m^{2}+m}}{\left(q^{3}, t ; q^{3}\right)_{m} q^{2 m}} \frac{\left(q^{3} ; q^{3}\right)_{m}\left(t ; q^{3}\right)_{m}\left(-z q ; q^{2}\right)_{m} \alpha^{m} q^{m}}{\left(q^{3} ; q\right)_{m}\left(-\alpha q^{2} ; q^{2}\right)_{m}} \\
& =\sum_{m=0}^{\infty} \frac{(t ; q)_{m}\left(-z q ; q^{2}\right)_{m} q^{m^{2}} \alpha^{m}}{\left(-\alpha q^{2} ; q^{2}\right)_{m}}=(t)_{\infty} S_{0}(t, \alpha, \beta, z ; q) .
\end{aligned}
$$


The left hand side of (6.3) is equal to

$$
\begin{gathered}
\sum_{k=0}^{\infty} \frac{\left(1-t q^{4 k-1}\right)\left(1-q^{-2 k+2}\right)\left(t / q, q^{2} ; q\right)_{k} q^{k^{2}+k}}{(1-t / q)\left(1-q^{2}\right)\left(q^{3}, t ; q^{3}\right)_{k} q^{2 k}} \\
\times \sum_{m=0}^{\infty} \frac{\left(t ; q^{3}\right)_{k}\left(t q^{3} ; q^{3}\right)_{m}\left(q^{3} ; q^{3}\right)_{k}\left(q^{3 k+3} ; q^{3}\right)_{m}\left(-z q ; q^{2}\right)_{k}\left(-z q^{2 k+1} ; q^{2}\right)_{m} \alpha^{m+k} q^{m+k}}{\left(q^{3} ; q\right)_{k}\left(q^{k+3} ; q\right)_{m}\left(-\alpha q^{2} ; q^{2}\right)_{k}\left(-\alpha q^{2 k+2} ; q^{2}\right)_{m}} \\
=\sum_{k=0}^{\infty} \frac{\left(1-t q^{4 k-1}\right)\left(1-q^{-2 k+2}\right)(t ; q)_{k-1}\left(-z q ; q^{2}\right)_{k} q^{k^{2}} \alpha^{k}}{\left(1-q^{k+2}\right)\left(-\alpha q^{2} ; q^{2}\right)_{k}} \\
\times \sum_{m=0}^{\infty} \frac{\left(t q^{3} ; q^{3}\right)_{m}\left(q^{3 k+3} ; q^{3}\right)_{m}\left(-z q^{2 k+1} ; q^{2}\right)_{m} \alpha^{m} q^{m}}{\left(q^{k+3} ; q\right)_{m}\left(-\alpha q^{2 k+2} ; q^{2}\right)_{m}} \\
=\frac{1}{(t)_{\infty}} \sum_{k=0}^{\infty} \frac{\left(1-t q^{4 k-1}\right)\left(1-k^{-2 k+2}\right)(t ; q)_{k-1}\left(-z q ; q^{2}\right)_{k} q^{k^{2}} \alpha^{k}}{\left(1-q^{k+2}\right)\left(-\alpha q^{2} ; q^{2}\right)_{k}} \\
\times \phi\left[\begin{array}{l}
q ;-z q^{2 k+1} ; t q^{3 k}, q^{3 k+3} \\
q^{k+3} ;-\alpha q^{2 k+2}: 0
\end{array}\right.
\end{gathered}
$$

which proves (i).

Proof of (ii): Take $a=t / q, b=q^{2}, p=q$ and

$$
\alpha_{m}=\frac{\left(q^{3} ; q^{3}\right)_{m}\left(t ; q^{3}\right)_{m}\left(-q^{2} / \alpha ; q^{2}\right)_{m} q^{4 m+2} \alpha^{m}}{\left(q^{3} ; q\right)_{m}\left(-q / z ; q^{2}\right)_{m+1} z^{m+1}} \text { in }
$$

Proof of (iii): Take $a=t / q, b=q^{2}, p=q$ and

$$
\alpha_{m}=\frac{\left(q^{3} ; q^{3}\right)_{m}\left(t ; q^{3}\right)_{m}\left(-z q ; q^{2}\right)_{m} q^{m} z^{2 m} \alpha^{m}}{\left(q^{3} ; q\right)_{m}\left(-\alpha q^{4} ; q^{4}\right)_{m}} \text { in }
$$

Proof of (iv): Take $a=t / q, b=q^{2}, p=q$ and

$$
\alpha_{m}=\frac{\left(q^{3} ; q^{3}\right)_{m}\left(t ; q^{3}\right)_{m}\left(-z q ; q^{2}\right)_{m} q^{m} z^{2 m} \alpha^{m}}{\left(q^{3} ; q\right)_{m}\left(\alpha q ; q^{2}\right)_{m}} \text { in }
$$

Proof of (v): Take $a=t / q, b=q^{2}, p=q$ and

$$
\alpha_{m}=\frac{\left(q^{3} ; q^{3}\right)_{m}\left(t ; q^{3}\right)_{m}\left(-z q ; q^{2}\right)_{m} q^{3 m} \alpha^{m}}{\left(q^{3} ; q\right)_{m}\left(-\alpha q^{2} ; q^{2}\right)_{m}} \text { in }
$$

Proof of (vi): Take $a=t / q, b=q^{2}, p=q$ and

$$
\alpha_{m}=\frac{\left(q^{3} ; q^{3}\right)_{m}\left(t ; q^{3}\right)_{m}\left(-q^{2} / \alpha ; q^{2}\right)_{m} q^{2 m} \alpha^{m}}{\left(q^{3} ; q\right)_{m}\left(-q / z ; q^{2}\right)_{m+1} z^{m+1}} \text { in }
$$

Proof of (vii): Take $a=t / q, b=q^{2}, p=q$ and

$$
\alpha_{m}=\frac{\left(q^{3} ; q^{3}\right)_{m}\left(t ; q^{3}\right)_{m}\left(-z q ; q^{2}\right)_{m} q^{3 m+1} z^{2 m} \alpha^{m}}{\left(q^{3} ; q\right)_{m}\left(-\alpha q^{2} ; q^{4}\right)_{m+1}} \text { in }
$$

Proof of (viii): Take $a=t / q, b=q^{2}, p=q$ and

$$
\alpha_{m}=\frac{\left(q^{3} ; q^{3}\right)_{m}\left(t ; q^{3}\right)_{m}\left(-z q ; q^{2}\right)_{m} q^{3 m+1} z^{2 m} \alpha^{m}}{\left(q^{3} ; q\right)_{m}\left(\alpha q ; q^{2}\right)_{m+1}} \text { in }
$$

By taking $\alpha=1, \beta=1$ and $z=1$ we have multibasic expansion of classical eighth order mock theta functions. 


\section{Special cases and Ramanujan's cubic continued fraction}

Proposition 2. We have the following special cases

$$
\begin{aligned}
& \text { (i) } U_{0}(0,-1,1,1 ; q)=\frac{f(-q,-q)}{\psi(-q)}, \\
& \text { (ii) } U_{0}(0,-1,1,1 ;-q)=\frac{f\left(-q^{2},-q^{2}\right)}{\psi(-q)}, \\
& \text { (iii) } U_{0}(0,-1,3,-1 ;-q)=\frac{f\left(-q,-q^{5}\right)}{\psi(-q)} \\
& \text { (iv) } U_{0}(0,-1,1,-1 ;-q)=\frac{f\left(-q^{3},-q^{3}\right)}{\psi(-q)} .
\end{aligned}
$$

P r o o f. Proof of (i): By definition we have

$$
U_{0}(t, \alpha, \beta, z ; q)=\frac{1}{(t)_{\infty}} \sum_{n=0}^{\infty} \frac{(t)_{n} q^{n^{2}-n+n \beta}\left(-z q ; q^{2}\right)_{n} \alpha^{n}}{\left(-\alpha q^{4} ; q^{4}\right)_{n}}
$$

put $t=0, \alpha=-1, \beta=1$ and $z=1$, therefore we have

$$
U_{0}(0,-1,1,1 ; q)=\sum_{n=0}^{\infty} \frac{(-1)^{n} q^{n^{2}}\left(-q ; q^{2}\right)_{n}}{\left(q^{4} ; q^{4}\right)_{n}}
$$

from [10, eq. (A.13), p. 171], we have

$$
\frac{f(-q,-q)}{\psi(-q)}=\sum_{n=0}^{\infty} \frac{(-1)^{n} q^{n^{2}}\left(-q ; q^{2}\right)_{n}}{\left(q^{4} ; q^{4}\right)_{n}}
$$

by (7.2) and (7.3), we get

$$
U_{0}(0,-1,1,1 ; q)=\frac{f(-q,-q)}{\psi(-q)}
$$

which proves (i).

Proof of (ii): Put $t=0, \alpha=-1, \beta=1, z=1$ and replace $q=-q$ in (7.1), we have

$$
U_{0}(0,-1,1,1 ;-q)=\sum_{n=0}^{\infty} \frac{q^{n^{2}}\left(q ; q^{2}\right)_{n}}{\left(q^{4} ; q^{4}\right)_{n}},
$$

from [10, eq. (A. 23), p. 172], we have

$$
\frac{f\left(-q^{2},-q^{2}\right)}{\psi(-q)}=\sum_{n=0}^{\infty} \frac{q^{n^{2}}\left(q ; q^{2}\right)_{n}}{\left(q^{4} ; q^{4}\right)_{n}},
$$

by (7.4) and (7.5), we get

$$
U_{0}(0,-1,1,1 ;-q)=\frac{f\left(-q^{2},-q^{2}\right)}{\psi(-q)},
$$

which proves (ii). 
Proof of (iii): Put $t=0, \alpha=-1, \beta=3, z=-1$ and replace $q=-q$ in (7.1), we have

$$
U_{0}(0,-1,3,-1 ;-q)=\sum_{n=0}^{\infty} \frac{q^{n^{2}+2 n}\left(-q ; q^{2}\right)_{n}}{\left(q^{4} ; q^{4}\right)_{n}},
$$

from [10, eq. (A. 52), p. 175], we have

$$
\frac{f\left(-q,-q^{5}\right)}{\psi(-q)}=\sum_{n=0}^{\infty} \frac{q^{n^{2}+2 n}\left(-q ; q^{2}\right)_{n}}{\left(q^{4} ; q^{4}\right)_{n}},
$$

by (7.6) and (7.7), we get

$$
U_{0}(0,-1,3,-1 ;-q)=\frac{f\left(-q,-q^{5}\right)}{\psi(-q)}
$$

which proves (iii).

Proof of (iv): Put $t=0, \alpha=-1, \beta=1, z=-1$ and replace $q=-q$ in (7.1), we have

$$
U_{0}(0,-1,1,-1 ;-q)=\sum_{n=0}^{\infty} \frac{q^{n^{2}}\left(-q ; q^{2}\right)_{n}}{\left(q^{4} ; q^{4}\right)_{n}},
$$

from [10, eq. (A. 53), p. 175], we have

$$
\frac{f\left(-q^{3},-q^{3}\right)}{\psi(-q)}=\sum_{n=0}^{\infty} \frac{q^{n^{2}}\left(-q ; q^{2}\right)_{n}}{\left(q^{4} ; q^{4}\right)_{n}},
$$

by $(7.9)$ and (7.10), we get

$$
U_{0}(0,-1,1,-1 ;-q)=\frac{f\left(-q^{3},-q^{3}\right)}{\psi(-q)}
$$

which proves (iv).

Remark 1. Dividing (7.8) by (7.11), we have

$$
\frac{U_{0}(0,-1,3,-1 ;-q)}{U_{0}(0,-1,1,-1 ;-q)}=\frac{f\left(-q,-q^{5}\right)}{f\left(-q^{3},-q^{3}\right)}=1+\frac{q+q^{2}}{1}+\frac{q^{2}+q^{4}}{1}+\frac{q^{3}+q^{6}}{1} \ldots
$$

which is Ramanujan's cubic continued fraction [2, (3.1.6), p. 86].

\section{Conclusion}

The advantage of the generalization presented in the paper is that by specializing the parameters we can obtain known functions which connects mock theta functions with continued fractions. So the results obtained for mock theta functions are reduced to continued fractions.

\section{Acknowledgement}

The author thanks the reviewers for their useful comments and Prof. Bhaskar Srivastava for his guidance. 


\section{REFERENCES}

1. Andrews G.E. On basic hypergeometric series, mock theta functions and partitions (I). Q. J. Math., 1966. Vol. 17, No. 2. P. 64-80. DOI: 10.1093/qmath/17.1.64

2. Andrews G. E., Berndt B.C. Ramanujan's Lost Notebook. Part I. New York: Springer-Verlag, 2005. 437 p. DOI: $10.1007 / 0-387-28124-\mathrm{X}$

3. Andrews G. E., and Hickerson D. Ramanujan's "lost" notebook VII: The sixth order mock theta functions. Adv. Math., 1991. Vol. 89, No. 1. P. 60-105. DOI: 10.1016/0001-8708(91)90083-J

4. Choi Y.-S. The basic bilateral hypergeometric series and the mock theta functions. Ramanujan J., 2011. Vol. 24. P. 345-386. DOI: 10.1007/s11139-010-9269-7

5. Collected Papers of Srinivasa Ramanujan. Hardy G.H., Seshu Aiyar P.V., Wilson B.M. (eds.) New York: Chelsea Pub. Co., 1962 (C)1927). 355 p.

6. Gasper G., Rahman M. Basic Hypergeometric Series. Cambridge: Cambridge University Press, 1990. $276 \mathrm{p}$.

7. Gordon B., MacIntosh R. J. Some eighth order mock theta functions. J. London Math. Soc., 2000. Vol. 62, No. 2. P. 321-335. DOI: 10.1112/S0024610700008735

8. Jackson F. H. Basic Integration. Q. J. Math., 1951. Vol. 2, No. 1. P. 1-16. DOI: 10.1093/qmath/2.1.1

9. Rainville E. D. Special Function. New York: Chelsea Pub. Co., 1960. 365 p.

10. Sills A. V. An Invitation to the Rogers-Ramanujan Identities. New York: Chapman and Hall/CRC, 2017. 256 p. DOI: $10.1201 / 9781315151922$

11. Srivastava B. A generalization of the eighth order mock theta functions and their multibasic expansion. Saitama Math. J., 2006/2007. Vol. 24. P. 1-13.

12. Watson G. N. The final problem: An account of the mock theta functions. J. London Math. Soc., 1936. Vol. 11. P. 55-80. DOI: $10.1112 /$ jlms/s1-11.1.55 